\title{
Radiation-Pressure Acceleration of Ion Beams from Nanofoil Targets: The Leaky Light-Sail Regime
}

\author{
B. Qiao, ${ }^{1,2}$ M. Zepf, ${ }^{2}$ M. Borghesi, ${ }^{2}$ B. Dromey, ${ }^{2}$ M. Geissler, ${ }^{2}$ A. Karmakar, ${ }^{1}$ and P. Gibbon ${ }^{1}$ \\ ${ }^{1}$ Jülich Supercomputing Center, Forschungzentrum Jülich GmbH, D-52425, Jülich, Germany \\ ${ }^{2}$ Department of Physics and Astronomy, Queen's University Belfast, Belfast BT7 1NN, United Kingdom
} (Received 31 March 2010; published 4 October 2010)

\begin{abstract}
A new ion radiation-pressure acceleration regime, the "leaky light sail," is proposed which uses subskin-depth nanometer foils irradiated by circularly polarized laser pulses. In the regime, the foil is partially transparent, continuously leaking electrons out along with the transmitted laser field. This feature can be exploited by a multispecies nanofoil configuration to stabilize the acceleration of the light ion component, supplementing the latter with an excess of electrons leaked from those associated with the heavy ions to avoid Coulomb explosion. It is shown by $2 \mathrm{D}$ particle-in-cell simulations that a monoenergetic proton beam with energy $18 \mathrm{MeV}$ is produced by circularly polarized lasers at intensities of just $10^{19} \mathrm{~W} / \mathrm{cm}^{2}$. $100 \mathrm{MeV}$ proton beams are obtained by increasing the intensities to $2 \times 10^{20} \mathrm{~W} / \mathrm{cm}^{2}$.
\end{abstract}

DOI: 10.1103/PhysRevLett.105.155002

Multi-MeV ion acceleration from laser-irradiated solid foils has become a highly active field of research over the past few years [1-6]. The wide potential applications [7] include tumor therapy, radiography, and laser-driven fusion. Most applications require a high-energy ion beam with large particle number and monoenergetic spectrum. Radiation-pressure acceleration (RPA) [3-6] using circularly polarized $(\mathrm{CP})$ laser pulses has emerged as a promising route to obtaining such high-quality ion beams in a much more efficient manner, compared to the target normal sheath acceleration (TNSA) [1,2].

In the simplest version of RPA scheme, the ion and electron layers are synchronously boosted by the laser pulse as if they constitute a quasineutral plasma slab, where equilibrium between the electrostatic and radiation pressures holds. The final ion peak energy is determined by the laser pulse energy and the slab total mass. By decreasing the foil thickness, enhancement in both the peak energy and conversion efficiency can be achieved due to the target's limited mass. Recently, the acceleration of highenergy ion beams from nanometer $(\mathrm{nm})$ foils has attracted particular interest $[8,9]$.

Depending on the foil thickness $l_{0}$, three regimes can be identified for the RPA scheme. First, if the foil is semiinfinite, ions experience a "hole-boring" (HB) phase of RPA $[10,11]$. At an early stage, the electron and ion density profiles and the electrostatic field $E_{z}$ before ions move are described by Fig. 1(a). Electrons pile up into a compressed layer of thickness $d_{2} \sim l_{s}$, where $l_{s}=c / \omega_{p e}$ is the foil skin depth of density $n_{e 0}$ and $\omega_{p e}=\sqrt{4 \pi n_{e 0} e^{2} / m_{e}}$. Since the HB is continuous, all ions are accelerated by $E_{z}$ in a pistonlike manner, finally reaching a maximum velocity $v_{i, \max }=2 v_{b}$, where $v_{b} / c=a /\left(2 \sqrt{m_{i} n_{i}}\right) ; m_{i}, n_{i}$, and $a$ are ion mass, density, and the normalized laser amplitude. To obtain high-energy ion beams a very high-intensity laser is
PACS numbers: 52.38.Kd, 29.25.-t, 52.50.Jm, 52.65.Rr

required as $v_{i, \max }$ does not increase with time after the $\mathrm{HB}$ has saturated.

Second, if the foil is of thickness $l_{0}>l_{s}$, typically from tens of $\mathrm{nm}$ to microns, electrons pile up at its rear side in a thin layer of thickness $d_{2} \ll l_{0}$, and are held inside because the laser field vanishes at the foil rear surface-Fig. 1(b). Only the ions initially located in the compression region $\left(d_{1}<z<l_{0}\right)$ at the rear side are bunched into a highdensity layer by the leading edge of $E_{z}$, while the others undergo Coulomb explosion by the trailing part of $E_{z}$ [12]. Afterwards, the electron and ion layers combine together as a quasineutral plasma slab pushed by the laser, known as "light-sail" (LS) RPA regime [13]. However, in a realistic multidimensional case, this plasma slab subsequently loses electrons due to transverse instabilities $[4,6,14]$, which quickly becomes positively charged. As a result, the ion beam debunches by a rapid Coulomb explosion and cannot be stably accelerated. Stable acceleration can be achieved only when the laser intensity $I_{0} \geq 10^{22} \mathrm{~W} / \mathrm{cm}^{2}[6]$.

When the foil is of nm-scale thickness, less than (or close to) its skin depth $l_{s}$, the laser field does not
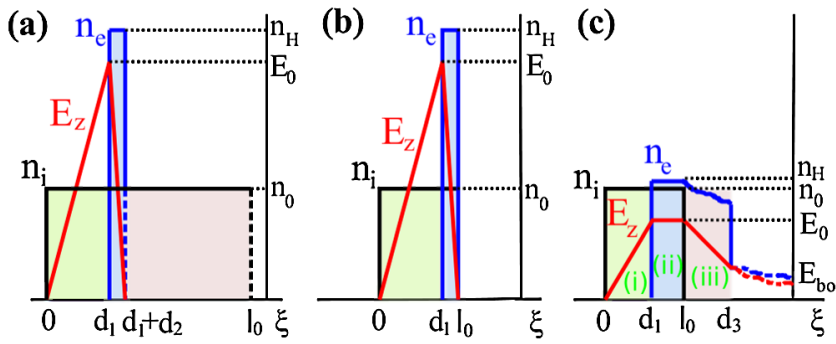

FIG. 1 (color online). Schematic profiles of ion $\left(n_{i}\right.$, black) and electron $\left(n_{e}\right.$, blue $)$ densities and of the electrostatic field $\left(E_{z}\right.$, red $)$ at an early stage of $\mathrm{CP}$ laser foil interactions before ions move. (a)-(c) are, respectively, for the foils in thickness of semiinfinite, $\mu \mathrm{m}$-scale, and $\mathrm{nm}$-scale cases. 
significantly decay within the foil and so all electrons will be accelerated by the transmitted field. Electrons near the rear side will leak into vacuum since the laser field does not vanish there. The equilibrium states depicted in Figs. 1(a) or 1(b) break down. Consequently, theoretical models of either "phase-stable" [5,9] or "cyclic" [4,12] acceleration no longer apply to this new regime, which we call "leaky light-sail (LS)" RPA. In this Letter, we present theoretical and numerical studies on this new regime, where a subskin-depth $\mathrm{nm}$ foil (nanofoil) is irradiated by CP laser pulses. A theoretical model is given, consistent with two-dimensional (2D) particle-in-cell (PIC) simulations. Exploiting the feature that the foil constantly leaks electrons from its rear, a multispecies nanofoil is suggested by which the light ions can achieve stable acceleration in a multidimensional geometry by moderate $\mathrm{CP}$ lasers. The idea is verified by $2 \mathrm{D}$ simulations with a hydrocarbon foil. A monoenergetic proton beam of energy $18 \mathrm{MeV}$ is produced by CP lasers at $I_{0}$ of $10^{19} \mathrm{~W} / \mathrm{cm}^{2}$. By increasing $I_{0}$ to $2 \times 10^{20} \mathrm{~W} / \mathrm{cm}^{2}, 100 \mathrm{MeV}$ proton beams are obtained.

We first run 2D PIC simulations for a single-species nanofoil to get an insight into this new regime using the code "ILLUMINATION." We choose a CP laser pulse with wavelength $\lambda=1.0 \mu \mathrm{m}$ and intensity $I_{0}=3.4 \times$ $10^{19} \mathrm{~W} / \mathrm{cm}^{2}\left(a_{0}=5\right)$. The pulse is incident along the $z$ axis from $z=0$ with flattop envelope of 1-cycle rise time and 40-cycle plateau. A fully ionized hydrogen foil with electron and proton densities $n_{e 0}=n_{i p 0}=200 n_{c}$ is located at $z_{0}=3 \mu \mathrm{m}$. The foil thickness $l_{0}=8 \mathrm{~nm}$ is less than $l_{s}=11.3 \mathrm{~nm}$ but thick enough to satisfy

$$
(1 / \pi)\left(n_{c} / n_{e 0}\right) a_{0} \lambda \leq l_{0}<l_{s}
$$

ensuring not all electrons are blown out $[6,12]$. The simulation space $(15 \times 12 \mu \mathrm{m})$ is composed of $30000 \times 3000$ cells along $z$ and $x$ directions. Each foil cell is filled with 800 quasiparticles.

Figs. 2(a)-2(d) show density maps of electrons $n_{e}$ and protons $n_{i p}$ at time $t=12.5,20,34$, and $80 \mathrm{fs}$. At an early stage-Fig. 2(a) — we see that all foil electrons are accelerated: electrons at the front side are pushed inward, leaving behind a depletion region; however, electrons at the rear side are dragged into vacuum due to the nonvanished laser field there, forming a leakage region; electrons are much less compressed than in the standard LS case. The longitudinal profiles of $n_{e}, n_{i p}$, and $E_{z}$ in Fig. 2(e) clearly correspond to the schematic Fig. 1(c) rather than 1(a) or 1(b). Three charge separation regions can be distinguished: the (i) depletion $\left(0<\xi<d_{1}\right)$, (ii) quasineutral $\left(d_{1}<\xi<l_{0}\right)$, and (iii) leakage $\left(l_{0}<\xi<l_{0}+d_{3}\right)$ regions, where $\xi=z-z_{0}$. The electrostatic field $E_{z}$ can be modeled as

$$
E_{z}= \begin{cases}E_{0} \xi / d_{1} & \text { if } 0<\xi<d_{1}, \\ E_{0} & \text { if } d_{1} \leq \xi \leq l_{0}, \\ E_{0}\left[1-\left(\xi-l_{0}\right) / d_{3}\right]+E_{b o}\left(\xi-l_{0}\right) / d_{3} & \text { if } l_{0}<\xi \leq l_{0}+d_{3},\end{cases}
$$

including the leading and trailing edges and the uniform part, where $E_{0}=4 \pi e n_{e 0} d_{1}$ is the maximum value of $E_{z}$. $E_{b o} \neq 0$ is the contribution induced by the electrons blown out at $\xi>l_{0}+d_{3}$. Note that the exact profile of $E_{z}$ obtained here cannot be seen in lower-resolution simulations such as in [9].
Figures 2(b)-2(d) show acceleration at later times. We see that only the protons in the quasineutral region are bunched into a thin layer by the uniform part of $E_{z}$, while those in the depletion region are debunched via Coulomb explosion by the trailing edge of $E_{z}$. Meanwhile, only the electrons in the quasineutral region
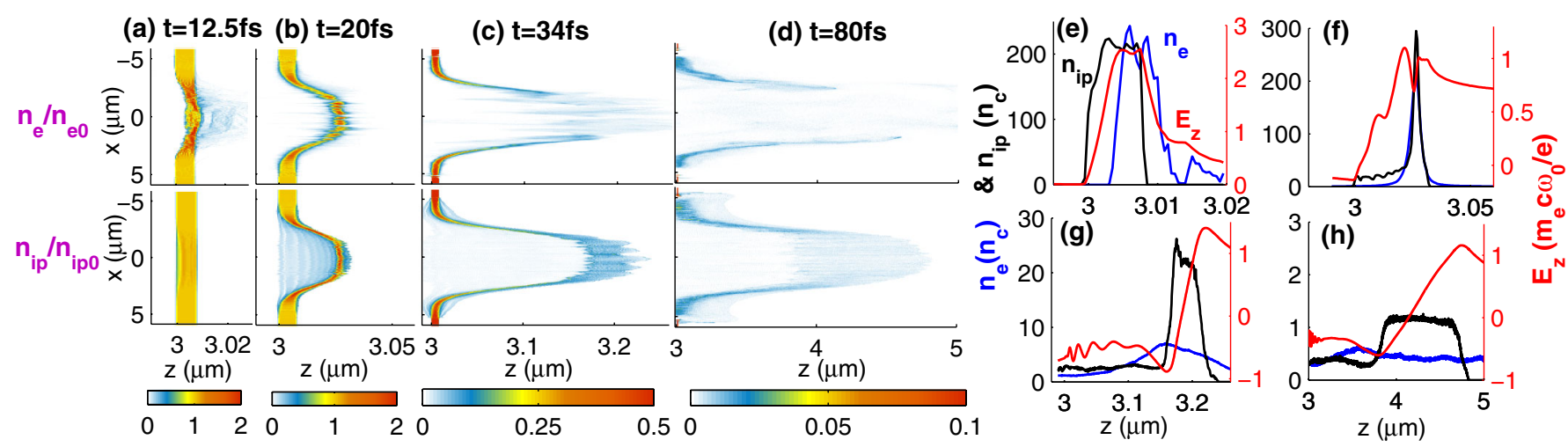

FIG. 2 (color online). (a)-(d) Electron $n_{e} / n_{e 0}$ and proton $n_{i p} / n_{i p 0}$ densities at $t=12.5,20,34$, and 80 fs for a hydrogen nanofoil with $n_{e 0}=n_{i p 0}=200 n_{c}$, and $l_{0}=8 \mathrm{~nm}$ irradiated by CP lasers at $I_{0}=3.4 \times 10^{19} \mathrm{~W} / \mathrm{cm}^{2}$. (e)-(h) are the corresponding longitudinal profiles of $n_{e}$ (blue), $n_{i p}$ (black), and the electrostatic field $E_{z}$ (red) cut at $x=0$. 
are accelerated as a bunched layer while those in the leakage region are blown out because no ions exist there. These ion and electron layers combine together forming a plasma slab of lower thickness $d_{2}<l_{0}<l_{s}$, see Fig. 2(b). Therefore, in contrast to the standard LS regime, this slab not only loses electrons at its laser-facing surface due to transverse instabilities but also leaks electrons from its rear due to the nonvanished laser field [2(b)]. The slab changes quickly from being negatively [2(f)] to positively [2(g) and 2(h)] charged. As soon as the slab is positively charged, the local $E_{z}$ acquires a pure trailing edge, ultimately resulting in the broad energy spectrum in Fig. 4(c) by the dashed line.

The above arguments imply that ion RPA in the Leaky LS regime is even less efficient than the standard LS regime if a single-species target is used. However, this negative result gives us a key insight of this new regime which may be turned into an advantage. The leakage of electrons from the target's rear may be useful for RPA of light ions (protons) in a multispecies nanofoil. It is obvious that a heavy ion with charge state $Z$ is accompanied by $Z$ times more electrons than a proton. If a multispecies nanofoil is used, the electrons leaked from those associated with the heavy ions may be acquired by the proton layer as a large negative charge supplement since proton only needs one electron for charge balance. As a result, despite the loss of electrons due to leakage and transverse instabilities, the proton layer may remain surrounded by excessive electrons, preventing Coulomb explosion and preserving stable RPA. Therefore, we expect protons from multispecies nanofoils in this regime to experience stable RPA, so that high-energy monoenergetic proton beams can be obtained by much lower-intensity lasers, compared to the standard LS case.

To check this idea by 2D simulations, we take a hydrocarbon $(\mathrm{CH})$ nanofoil and assume it to be fully ionized into
$\mathrm{C}^{6+}$ and $\mathrm{H}^{+}$. In order to maintain $l_{s}$ identical as in the single-species case, we choose the same electron density $n_{e 0}=200 n_{c}$ and thickness $l_{0}=8 \mathrm{~nm}$. The densities of $\mathrm{H}^{+}$ (proton) and $\mathrm{C}^{6+}$ are taken, respectively, as $n_{i p 0}=4.1 n_{c}$ and $n_{i c 0}=32.65 n_{c}$ with their ratio $n_{i p 0}: n_{i c 0} \approx 1: 8$. All the laser and other parameters are also the same.

Figs. 3(a)-3(h) give density maps and longitudinal profiles of electrons $n_{e}, \mathrm{C}^{6+}$ ions $n_{i c}$, and protons $n_{i p}$ at $t=$ $12.5,20,34$, and $80 \mathrm{fs}$. The early stages of $n_{e}$ and $E_{z}$ in (a) and (e) are very similar to those in the single-species case, presenting the same three typical regions. The multispecies effect does not influence the electron dynamics.

Similarly, we see that only the protons and $\mathrm{C}^{6+}$ ions initially in the quasineutral region are bunched into thin layers by the quasiuniform part of $E_{z}$ [Fig. 3(e)]. Protons having larger charge-mass ratio tend to move faster than the heavy $\mathrm{C}^{6+}$, so the proton layer moves ahead of the $\mathrm{C}^{6+}$ layer. If the accompanying electron layer is stably pushed without debunching or leakage, and equilibrium between the electrostatic and radiation pressures holds as in the $\mathrm{HB}$ regime [11], we know that the faster proton (slower $\mathrm{C}^{6+}$ ) layer will be pulled back (forward) by the space-charge redistribution and finally the entire slab (the proton, $\mathrm{C}^{6+}$ and electron layers) will move together at the same velocity $2 v_{b}$, which only depends on the total mass of the slab, irrespective of their individual charge and mass.

However, in the leaky LS regime the laser field does not significantly decay within the slab, which cannot be balanced by the electrostatic field. The electron layer debunches and constantly leaks electrons [Figs. 3(b) and 3(f)]. This debunching of electrons leads to an almost complete separation of the $\mathrm{C}^{6+}$ and proton layers; also see 3(b) and 3(f).

Moreover, due to the loss of electrons, the $\mathrm{C}^{6+}$ layer has insufficient electrons for charge balance and the local space charge becomes positive [Fig. 3(g)]. By contrast,
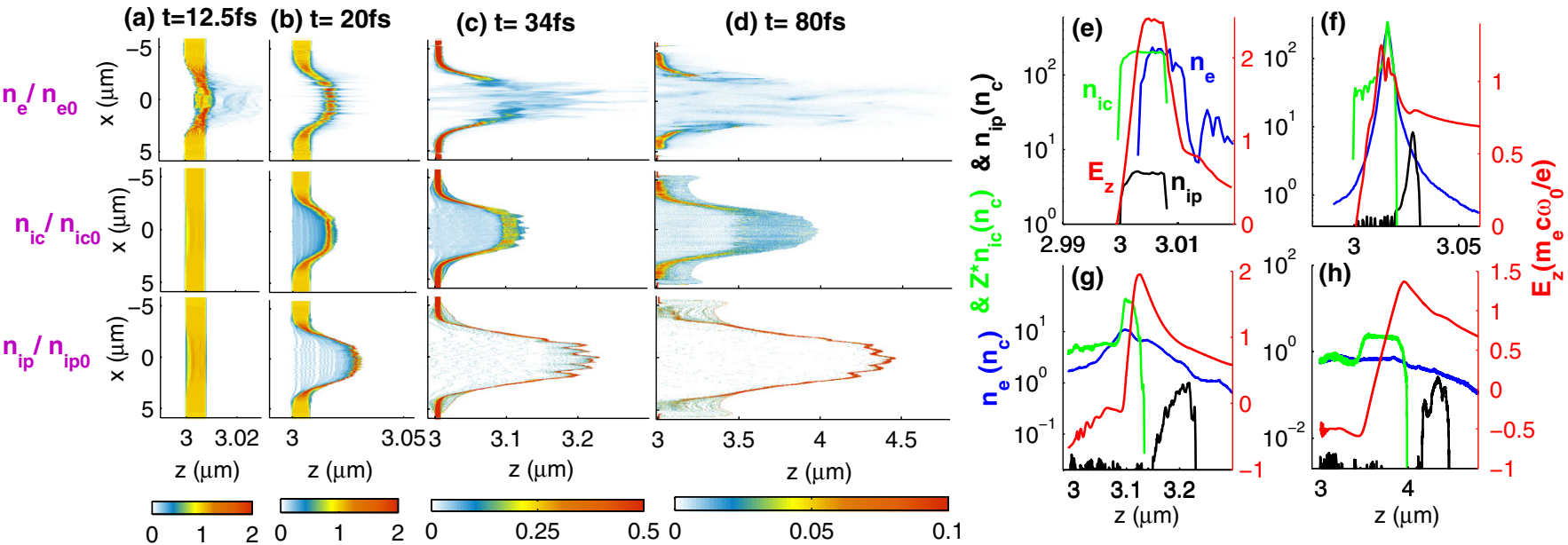

FIG. 3 (color online). (a)-(d) Electron $n_{e} / n_{e 0}, \mathrm{C}^{6+} n_{i c} / n_{i c 0}$ and proton $n_{i p} / n_{i p 0}$ densities at $t=12.5,20,34$, and $80 \mathrm{fs}$ for a $\mathrm{CH}$ nanofoil with $n_{e 0}=200 n_{c}, n_{i p 0}: n_{i c 0}=1: 8$, and $l_{0}=8 \mathrm{~nm}$ by CP lasers at $I_{0}=3.4 \times 10^{19} \mathrm{~W} / \mathrm{cm}^{2}$; (e)-(h) are the corresponding longitudinal profiles of $n_{e}$ (blue), $Z * n_{i c}=6 n_{i c}$ (green), $n_{i p}$ (black), and $E_{z}$ (red). 
the proton layer is surrounded by excessive electrons keeping a locally negative space charge [also $3(\mathrm{~g})]$ due to the supplementary of electrons leaked which were originally associated with the $\mathrm{C}^{6+}$ layer. In other words, the proton layer poaches electrons from the $\mathrm{C}^{6+}$ layer. Therefore, the proton layer maintains stable RPA by the leading edge of $E_{z}$ while the $\mathrm{C}^{6+}$ layer undergoes rapid Coulomb explosion by the trailing part of $E_{z}$ [see 3(d) and 3(h)].

Figure 4(a) shows a quasimonoenergetic proton beam with density about $0.25 n_{c}$ and peak velocity $0.18 c$ is obtained at $t=130 \mathrm{fs}$. The energy spectra of protons and $\mathrm{C}^{6+}$ are shown in 4(b). The proton beam has peak energy $18 \mathrm{MeV}$ with FWHM of $5 \mathrm{MeV}$ and particle number about $10^{8}$. The variations with time $t$ of the peak $E_{z}$ acting on the proton beam and the peak proton energy are given in 4(c) and 4(d). We can observe the typical features of the RPA scheme, $E_{z}$ decreases very slowly with $t$ as it is in balance with the radiation pressure; and the peak proton energy at a later time scales as $t^{1 / 3}$, consistent with the typical analytical scaling of RPA $[4,6]$. This proves that the proton beam does experience stable RPA rather than direct Coulomb explosion (DCE) [15]. As a matter of fact, the DCE scheme [15] has been proposed for the case of linearly polarized lasers, there the intensity must be extremely high of $10^{22} \mathrm{~W} / \mathrm{cm}^{2}$ so that almost all electrons are blown out and the ion core is accelerated by Coulomb explosion, contrary to the condition (1) described in this Letter.

Note that the proton acceleration time here is determined by the laser pulse duration. For a laser pulse with finite duration $\tau_{L}$, the upper limit of proton energy can be estimated as $2 \eta^{2} \mathcal{E}_{L}^{2} /\left(2 \eta \mathcal{E}_{L}+\mathcal{N}_{i} m_{i} c^{2}\right) \mathcal{N}_{i}$, where $\mathcal{E}_{L}=$ $I_{0} S \tau_{L}$ is the laser energy, $S$ is its transverse area; $\mathcal{N}_{i}$ and $\eta$ are the particle number and conversion efficiency. One can therefore obtain higher-energy proton beams, such that
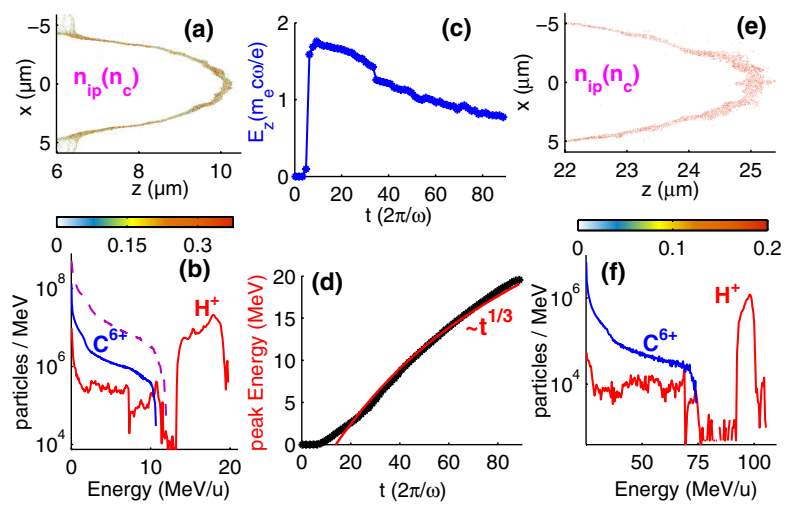

FIG. 4 (color online). (a) Density map and (b) energy spectrum of the proton beam obtained at $t=130 \mathrm{fs}$ in the acceleration of Fig. 3; (c) and (d) are the variations with time of, respectively, the peak $E_{z}$ and the peak proton energy. (e) and (f) are density map and energy spectrum of $100 \mathrm{MeV}$ proton beams obtained at $t=230 \mathrm{fs}$ by lasers of $I_{0}=2 \times 10^{20} \mathrm{~W} / \mathrm{cm}^{2}$, other parameters see the text. The dashed line in (b) is the spectrum for the singlespecies case in Fig. 2.
$100 \mathrm{~s}$ of $\mathrm{MeV}$, by using a CP laser of longer pulse duration and intensity experimentally accessible with existing laser systems. For example, we choose a CP pulse with 70-cycle flattop and intensity $2 \times 10^{20} \mathrm{~W} / \mathrm{cm}^{2}$ to irradiate on a CH nanofoil of $l_{0}=6.4 \mathrm{~nm}$ and $n_{e 0}=600 n_{c}$, with the condition (1) satisfied. The density ratio of $\mathrm{H}^{+}$to $\mathrm{C}^{6+}$ is $1: 12$. Figures 4(e) and 4(f) show a monoenergetic proton beam of energy $100 \mathrm{MeV}$ and density $0.15 n_{c}$ is obtained. The particle number is about $10^{7}$.

To summarize, a new "leaky light-sail" regime of ion RPA from sub-skin-depth nanofoils by CP laser pulses has been proposed. The key characteristic of this regime is that the foil constantly leaks electrons from its rear, meaning that a multispecies nanofoil can be used to achieve stable RPA of the light ions at moderate laser intensities. Twodimensional PIC simulations show that a monoenergetic proton beam of energy $18 \mathrm{MeV}$ is produced by irradiation of a $\mathrm{CH}$ nanofoil with $\mathrm{CP}$ lasers at intensities $3.4 \times$ $10^{19} \mathrm{~W} / \mathrm{cm}^{2}$, whereas $100 \mathrm{MeV}$ proton beams are obtained at intensities $10^{20} \mathrm{~W} / \mathrm{cm}^{2}$, experimentally available with the existing laser system. The high contrast in excess of $10^{10}$ is required for the laser, which can be achieved by using either plasma mirrors or parametric amplifications [16].

B. Q. acknowledges the support from the Alexander von Humboldt Foundation. M.Z. thanks the support from the Royal Society. The work was also supported by EPSRC (Grants No. EP/C003586/1 and No. EP/D/06337X/1). Computing resources on JUROPA at JSC (Grants No. jzam04 and No. jjsc04) and VIP at RZG (collaboration grant with MPQ) are both acknowledged.

[1] E. L. Clark et al., Phys. Rev. Lett. 84, 670 (2000); A. Maksimchuk et al., ibid. 84, 4108 (2000); R. A. Snavely et al., ibid. 85, 2945 (2000).

[2] B. M. Hegelich et al., Nature (London) 439, 441 (2006); H. Schwoerer et al., Nature (London) 439, 445 (2006).

[3] A. Macchi et al., Phys. Rev. Lett. 94, 165003 (2005).

[4] A. P. L. Robinson et al., New J. Phys. 10, 013021 (2008).

[5] X. Q. Yan et al., Phys. Rev. Lett. 100, 135003 (2008).

[6] B. Qiao et al., Phys. Rev. Lett. 102, 145002 (2009).

[7] M. Borghesi et al., Fusion Sci. Technol. 49, 412 (2006).

[8] A. Andreev et al., Phys. Rev. Lett. 101, 155002 (2008).

[9] A. Henig et al., Phys. Rev. Lett. 103, 245003 (2009).

[10] A. P. L. Robinson et al., Plasma Phys. Controlled Fusion 51, 024004 (2009).

[11] A. P. L. Robinson et al., Plasma Phys. Controlled Fusion 51, 095006 (2009).

[12] A. Macchi, S. Veghini, and F. Pegoraro, Phys. Rev. Lett. 103, 085003 (2009).

[13] J. F. L. Simmons and C. R. Mcinnes, Am. J. Phys. 61, 205 (1993).

[14] F. Pegoraro and S. V. Bulanov, Phys. Rev. Lett. 99, 065002 (2007).

[15] S. S. Bulanov et al., Phys. Rev. E 78, 026412 (2008).

[16] B. Dromey et al., Rev. Sci. Instrum. 75, 645 (2004); F. Tavella et al., Opt. Express 14, 12822 (2006). 